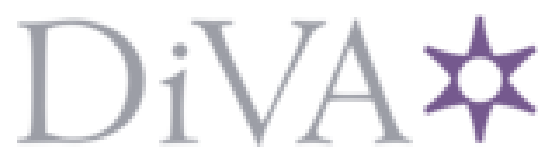

http://www.diva-portal.org

This is the published version of a paper published in Composites Science And Technology.

Citation for the original published paper (version of record):

Kaufmann, M., Zenkert, D., Mattei, C. (2008)

Cost optimization of composite aircraft structures including variable laminate qualities.

Composites Science And Technology, 68(13): 2748-2754

http://dx.doi.org/10.1016/j.compscitech.2008.05.024

Access to the published version may require subscription.

N.B. When citing this work, cite the original published paper.

Permanent link to this version:

http://urn.kb.se/resolve?urn=urn:nbn:se:kth:diva-11473 


\title{
Cost optimization of composite aircraft structures including variable laminate qualities
}

\author{
Markus Kaufmann $^{a}$, Dan Zenkert ${ }^{a}$ and Christophe Mattei ${ }^{b}$ \\ ${ }^{a}$ Kungliga Tekniska Högskolan (KTH), \\ 10044 Stockholm, Sweden \\ ${ }^{b}$ Division of Engineering Materials, \\ Department of Management and Engineering, \\ Linköpings Universitet, 58183 Linköping, Sweden
}

February 2008

Composite structures can lower the weight of an airliner significantly. The increased production cost, however, requires the application of costeffective design strategies in which cost, weight and the desired laminate quality are taken into account. This paper proposes an optimization framework for composite aircraft structures that minimizes the direct operating cost on a part level. In addition to previous models, a nondestructive testing model is implemented that calculates design allowables of a laminate based on ultrasonic scan parameters. In a case study, the effect of the laminate quality on the direct operating cost is discussed. It is investigated how the permissible flaw size and therefore the scan pitch of a composite laminate can influence the optimal solution in terms of cost and weight; thus, the manufacturing cost, the non-destructive testing cost and the weight of a component can be balanced by optimizing the laminate quality in an early design phase.

Keywords: Structural Composites, Non-destructive testing, Strength, Finite element analysis (FEA), Optimization

\section{Introduction}

Today's performance requirements necessitate the full application of carbon fibers to primary structures of airliners. The shift to composite materials lowers the structural 
weight significantly. The drawback, however, is the increased manufacturing cost. Through cost-effective design, tradeoffs between the minimum weight and the minimum cost solutions are found, two extremes that often contradict each other. Weight savings are directly associated with reduced fuel consumption and increased payload, while the reduced manufacturing cost has an impact on the acquisition cost. Therefore, the focus should not only be on pure weight reduction, but rather on a combined minimization of manufacturing cost and structural weight.

Attempts for a combined cost/weight optimization have been done by Geiger and Dilts [1], Heinmuller and Dilts [2], Kassapoglou [3-5], Marx et al. [6], Gantois [7], the Rolls-Royce's DATUM project [8], Park et al. [9], Edke and Chang [10], and Curran et al. [11]. In particular, Kelly and Wang [12], Wang et al. [13], Curran et al. [14], and Kaufmann et al. [15] incorporated the idea of a weight penalty; this weight penalty was applied to balance the tradeoff between manufacturing cost and structural weight. Each kilogram of structural weight was given a lifetime fuel burn cost, and the sum of fuel burn cost and manufacturing cost formed the objective function. Apart from embedding cost and weight, not much research has been performed on optimizing aircraft structures for life-cycle cost (LCC) or direct operating cost (DOC), the latter defined by Roskam [16]. Further, no attempts have been done to include the material quality of composite laminates into these optimization frameworks.

In the studies cited above it was assumed that the quality of the raw material was consistent and that no isolated inspection cost was identified in the cost models. This is due to the fact that the models were built up for metallic structures where in-service inspection plays a minor role in the design process, or that the cost model was not detailed enough to capture these costs. Unlike their metallic counterparts, however, composite parts are fabricated in-situ and the quality of these structures is highly dependent on the process robustness and the workmen skills. As a result, every composite part needs rigorous ultrasonic testing. Non-destructive testing (NDT) prevails as the prime means to maintain the quality assurance of structures. Typical manufacturing-generated defects in composite parts are voids, porosity, fiber misalignment, wrinkling, poor cure, resin-rich or resin-poor areas, the inclusion of foreign objects or contamination

Both the manufacturing of autoclave prepreg parts and the NDT methods have continuously been developed and refined during the last years. Nevertheless, the design is still based on rather conservative presuppositions, such as two percent through-the-thickness porosity and flaws of a size of typically $6 \mathrm{~mm}$ in diameter. In this work, these presuppositions are challenged. In particular, the optimization framework developed in [15] was enhanced by an NDT cost and strength reduction model.

The first time NDT cost was mentioned in the literature was in 1937 by Schmid [17]. There, x-ray, gamma and magnetic testing were investigated in terms of recurrent and non-recurrent costs. Although the test methods and components were not related to aircraft structures, this article is noteworthy. Schmid pointed out that the choice of an appropriate testing method has to be in proportion to its cost, the 
cost of the structural part and the cost of possible flaws and damages. Depending on the complexity of the part, different technical skills were needed, leading to different labor cost. Simple, similar components, for example, need a less qualified operator than complex, forged components. The cost was further determined by the inspection method, the part size and the reliability in flaw detection.

Since then, most of the relevant literature concentrated on the cost of aircraft maintenance and not on in-production testing. For instance, the life-cycle cost of aircrafts under the consideration of NDT was addressed by Hagemaier [18, 19]. Another example was shown by Kale [20] who combined the weight optimization of an aluminum panel with a reliability based model of the periodic in-service inspection.

In this work, a cost/weight optimization framework is proposed that incorporates manufacturing cost, inspection cost and the weight of the component. In addition to previous studies, the NDT cost is calculated separately by means of a feature-based cost estimation model. Moreover, the laminate quality is considered as a design variable; this variable has a distinct influence on the scan parameters and therefore the scan cost, and it controls the strength prediction of the laminate.

\section{Method}

The optimization framework herein consists of the necessary routines to calculate the objective function and the constraints. In the following these blocks are described in detail.

\subsection{Optimization framework}

Our problem for the combined optimization of manufacturing cost, NDT cost and weight is formulated as

$$
\begin{aligned}
\min & D O C \text { of a composite element } \\
\text { subject to } & \text { structural requirements } \\
& \underline{x}_{i}<x_{i}<\bar{x}_{i}, \quad i=1 \ldots n,
\end{aligned}
$$

where $x_{i}$ are the design variables and $\underline{x}_{i}$ and $\bar{x}_{i}$ the corresponding lower and upper limits. Examples of variables are laminate thicknesses, the distance between two adjacent stiffeners, the height and width of the stiffeners or the laminate quality.

The objective function of the optimization problem is formed by a simplified version of the direct operating cost $D O C$. Here, the $D O C$ is formed by the weighted sum

$$
D O C=\alpha_{1} C_{m a n}+\alpha_{2} C_{n d t, p r o d}+\alpha_{3} N C_{n d t, s e r v}+p W
$$

where $C_{\text {man }}$ is the manufacturing cost, $C_{n d t, p r o d}$ is the cost for non-destructive in-production testing, $C_{n d t, s e r v}$ is the cost of a single in-service inspection, $p$ is a 
weight penalty (given as the lifetime fuel burn cost per unit weight) and $W$ is the weight of the structure. The parameters $\alpha_{i}$ incorporate calibration factors due to depreciation, overhead cost and other cost adjustments, and $N$ is the number of regular inspections during the lifetime of the aircraft. In a first step this number is a constant, whereas in a more thorough analysis it could be based on the location, the stress level and the exposure of the part.

In Figure 1, the optimization routine is illustrated, including an analysis block containing an FE model, a manufacturing cost model, a weight model and an NDT model.

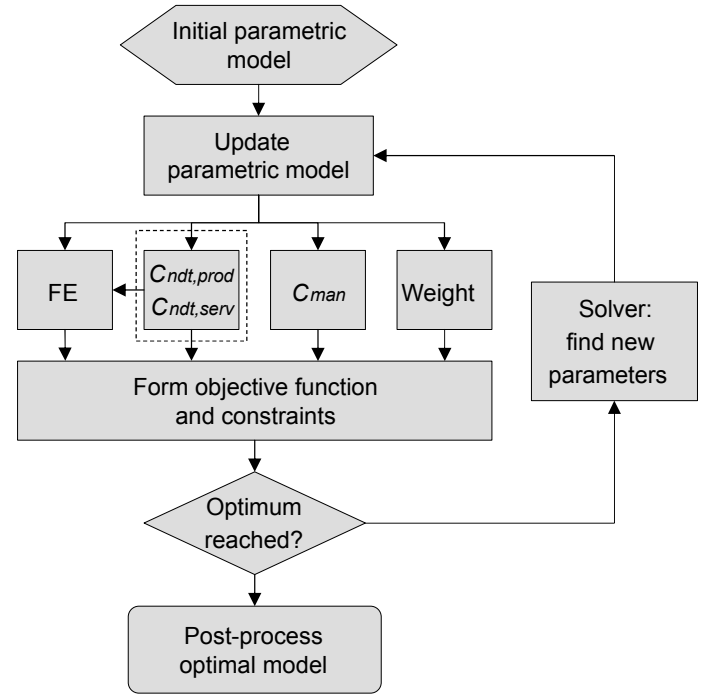

Figure 1: The optimization loop; the module within the dashed line is subject to further explanation below.

\section{$2.2 \quad$ FE analysis}

The FE evaluation is done in ABAQUS/Standard and comprises a linear buckling analysis and the computation of a composite failure criterion. It is important to note that the results and conclusions presented here are specific to the flaw type and size included in the analysis and to the failure modes assumed (buckling and maximum strain failure). They cannot be generalized before other failure modes (e.g. crippling, local buckling of a delamination, impact damage) are accounted for. Hence, the structural constraints used herein are only examples of possible criteria. The modular design of the optimization framework obviously allows for the implementation of more constraints than used in this analysis. 
The FE model is parameterized in terms of the variables $x_{i}$. Each change of a variable value results in a new set of buckling eigenvalues and maximum strain numbers, which form the structural constraints to the optimization problem. Python scripts are used for the parametrization.

\subsection{Manufacturing cost}

The manufacturing operations and processes of composites can be related to costs. Examples are the stacking of prepreg plies, debulking, consumables, curing or tooling. Each process represents material cost and a time need which can be translated into labor costs. The sum of all cost plus an overhead represents the total cost to manufacture the panel. This kind of feature-based model (see Niazi et al. [21]) is easily parameterizable, as features can be added, removed or modified. The commercially available cost estimation software SEER-MFG ${ }^{1}$ incorporates all these capabilities and is used therefore. Similar to the approach taken for the FE calculation, an initial model is built up in the graphical user interface of the software. This model contains all the necessary assumptions and work steps of the manufacturing process. It is exported to a text file, parameterized in terms of the variables $x_{i}$ and prepared for running in command line mode, the so-called server mode.

\subsection{Weight estimation}

The estimation of the weight is done by a simple calculation of the part volume in any given iteration, based on the CAD geometry, the material properties and the actual variables $x_{i}$.

\subsection{Non-destructive testing}

Unlike the estimation of the manufacturing cost, it was decided to develop an in-house feature-based tool for the evaluation of the non-destructive testing cost. It involves the estimation of the NDT cost for in-production and in-service testing, and the adjustment of the material strength.

Some preconditions that apply to the manufacturing of composite aircraft structures are assumed. For example, all components are thoroughly tested before the assembly, which implies that the total surface of each structural member is scanned. Testing is performed assuming conditions of series production, i.e. the testing process is cost optimal in terms of operator level and test equipment, and no non-recurring costs (such as the purchase of probes or other equipment) are incorporated. No reject rate was implemented - a reliability-based cost model could be developed in a later stage. The generic database provides the following features:

\footnotetext{
${ }^{1}$ see http://www.galorath.com
} 
- Thin flat laminate with access from both sides

- Thin flat laminate with access from one side

- Thick flat laminate with access from both sides

- Thick flat laminate with access from one side

- Radius

- Adhesive bond

Each of these features requires input data in form of the length $l_{k}$, the width $w_{k}$, the thickness $t_{k}$ and - in case of radii - the radius $r_{k}$, see Figure 2. On the other hand, the feature definition includes a scanning technique, a complexity index, the educational level of the operator and (associated with the latter) a cost per hour or per scanned area.

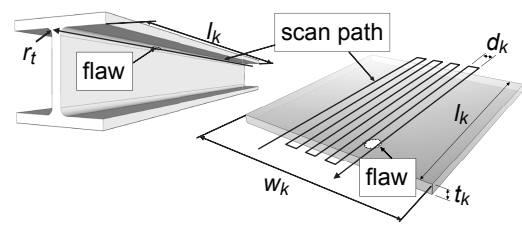

Figure 2: Ultrasonic C-scan of radii and flat plates

For rectangular scans of plates, flanges and adhesive bonds, the NDT cost of the $k^{t h}$ feature is calculated as

$$
C_{n d t, k}=\frac{l_{k} w_{k}}{v_{k} d_{k}} \cdot\left[C_{\text {machine }, k}+C_{\text {operator }, k}\right]
$$

where $v_{k}$ is the scan speed and $d_{k}$ is the scan pitch (distance between two scan paths) for the feature $k$. The parameters $C_{\text {machine, } k}$ and $C_{\text {operator }, k}$ represent the hourly rate of the test equipment and the operator, respectively.

For linear scans of radii, the NDT cost is calculated as

$$
C_{n d t, k}=\frac{l_{k}}{v_{k}} \cdot\left[C_{\text {machine }, k}+C_{\text {operator }, k}\right]
$$

and the total cost of non-destructive testing is the sum of all features

$$
C_{n d t}=\sum_{k=1}^{M} C_{n d t, k} \quad M=\text { Number of features. }
$$

Apart from the scanning cost per area, one has to consider a dependency of the cost on the laminate thickness. Several approaches of how to tackle this problem were examined. Finally, it was decided to introduce a method of adjusting the structural allowable due to higher risk of porosity or flaws in thicker structures. Hence, a relationship between testing cost and weight is found through the quality of the laminate. The following two reductions of the material strength are evaluated. 
- Strength $\hat{\sigma}_{p, k}$ due to possible porosity; a function of the thickness and the material of the cross-section.

- Strength $\hat{\sigma}_{f, k}$ due to possible flaws or foreign objects; a function of the scan pitch, the thickness, the material properties (based on coupon tests) and the NDT equipment.

Note that all strengths are calculated feature-wise. The strength that is applied in the FE calculation is the minimum of each $\hat{\sigma}_{p, k}$ and $\hat{\sigma}_{f, k}$. Both mechanisms are described below.

\subsection{Strength reduction due to porosity}

In the design of composite structures, a certain level of porosity is tolerated and taken into account in the calculation of the structural performance. Typically, an acceptance level of two volume percents is stated, a level that has been kept constant during the last two decades. With regard to new material systems, optimized manufacturing methods and more accurate NDT techniques, this porosity level is about to be challenged.

A lot of work has been performed to investigate in the effect of the laminate thickness and the laminate porosity on the mechanical properties [22-27]. It was concluded that the compressive strength $\hat{\sigma}_{c}$ degrades exponentially. In the context of isotropic materials, the equation

$$
\hat{\sigma}=\hat{\sigma}_{\max } e^{B v_{p}}
$$

has elevated to describe this behavior, where $\hat{\sigma}_{\max }$ is the strength at zero porosity, $v_{p}$ is the volume fraction of pores and $B$ is an empirical constant that depends on orientation, shape and size of the pores.

As the proposed method is a part of the design process, the laminate thickness $t$ has to be translated to an anticipated porosity level $v_{p}$ for a given material and manufacturing technique. Lee and Soutis [26] performed a study on the void content with respect to the thickness of the specimen and concluded that this value is highly dependent on the stacking sequence of the laminate.

\subsection{Strength reduction due to foreign objects}

All manufactured composite parts have to be tested for the inclusion of foreign objects (e.g. parts of the release paper of the prepreg) and delaminations. Nowadays, the composite manufacturer defines a minimum detectable flaw size, typically being around $6 \mathrm{~mm}$ in diameter.

The probability of detection never reaches $100 \%$. Instead, confidence levels based on the material, the NDT method and the thickness of the material are used; quoted levels are $90 \%$ or $95 \%$, see Georgiou [28]. Typical stochastic models used are the 
Gamma distribution or the two-parameter Weibull distribution, see Huang et al. [29]. For the Weibull distribution, the cumulative distribution function of the probability of detection $(\mathrm{POD})$ is given as

$$
P O D=1-e^{-(f / \lambda)^{k}}
$$

where $f$ is the flaw size, and $\lambda$ and $k$ are two shape parameters. A typical curve plot for different settings of $\lambda$ is shown in Figure 3 .

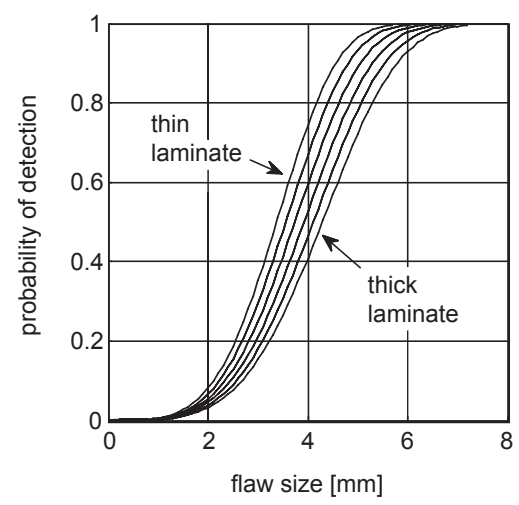

Figure 3: Probability of Detection vs. maximum flaw size and laminate thickness

As can be seen in this figure, the $P O D$ is higher for thinner laminates (the actual value depends on the material, the stacking sequence and the NDT method) and for larger flaw diameters. Today's $6 \mathrm{~mm}$ flaw diameter would require a scan pitch $d_{k}$ of approximately $2 \mathrm{~mm}$, the latter having a direct influence on the NDT costs. By varying the permissible flaw size, the quality of the laminate becomes a design variable and the material design strength is affected accordingly.

Different ways to relate the strength to the flaw size exist. Here, open hole compression is proposed, as it represents the most conservative strength reduction for a flaw of a certain size. For examples on the strength reduction of notched laminates, see Soutis et al. [30]. Other options could include a separate strength prediction model in FE (e.g. the simulation of a compression-after-impact coupon test) which takes into account the actual stacking sequence and the flaw size of the section, or the implementation of local delamination buckling as a function of the flaw size and the through-thickness location of the flaw.

The variable quality level can be explained as follows: Assuming that an allowed flaw diameter $f$ of less than $6 \mathrm{~mm}$ is applied causes all flaws bigger than $f$ to be detected. This leads to higher NDT cost and design strength, and to lower structural weight and manufacturing cost. A permissible flaw diameter $f$ of more than $6 \mathrm{~mm}$ leads to lower NDT cost and design strength, and to higher structural weight and manufacturing cost due to the additional plies needed. 
The algorithm is shown in Figure 4. As can be seen, the thickness $t$ and the maximum allowed flaw size $f$ form the input to the NDT module. In a first step, the scan pitch $d_{k}$ is adapted in order to reach the desired probability of detection. The direct cost for non-destructive testing $C_{n d t}$ is a function of the scanned area (length $l$ and width $w$ ) and the scan pitch $d_{k}$. Simultaneously, the strength reduction is calculated from the permissible flaw size and fed into the FE module where the constraints for the optimization are computed.

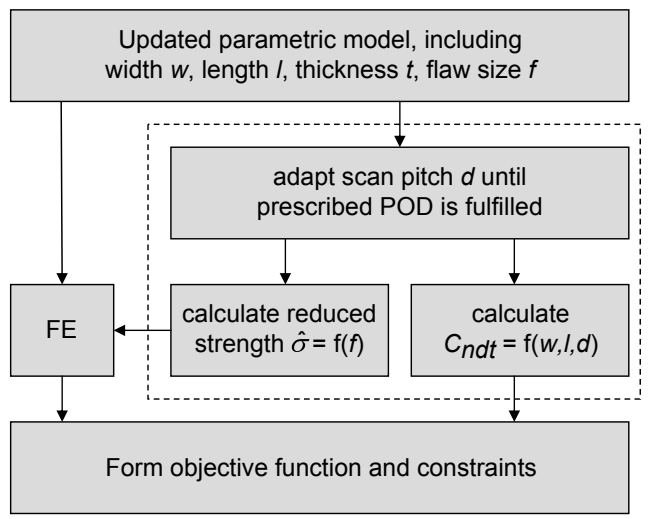

Figure 4: Flow chart of the strength reduction and cost calculation mechanism for non-destructive testing of one single feature

\subsection{Solver}

Based on the results of the analysis, the objective function and the optimization constraints are evaluated. Then the solver calculates adjusted variables and triggers the execution of the next iteration. For that purpose, a solver was sought that would incorporate the analysis blocks in the form of input-output models, not being too sensitive to disturbances in the form of non-smooth objective and constraint functions, and thus leading to a good convergence rate. In order to minimize the number of FE evaluations, the method of moving asymptotes (MMA) was chosen, see Svanberg $[31,32]$. The parametrization of the design variables as continuous variables is an idealization necessary to accommodate the use of this gradient-based method.

In reality, the thickness is not a continuous variable and it is possible that a gradient-based method will miss some designs that may be more efficient than the ones obtained from the optimization. Hence, the results from the present study are representatives of the trends and relative differences of alternate designs but may not necessarily spot the "best" solution. 


\section{Case study}

In a case study, a generic skin/stringer element is optimized in order to show the influence of the NDT cost and the quality level on the overall design. The panel (seen in Figure 5) consists of a laminated composite skin and two I-shaped stiffeners that are adhesively bonded to the skin. As can be seen the skin/stringer element is parameterized in terms of continuous variables, such as the stringer pitch, the panel thickness, the profile thickness, the web height and the flange width. The discrete stacking stacking sequence $\left[0_{m} / 90_{n} / \pm 45_{p}\right]_{s}$ was simplified to a $[0 / 90 / \pm 45]_{s}$ layup with continuous thickness variables. Note that the laminate generally consists of $70 \%$ fibers in the 0 degree, $20 \%$ in the \pm 45 degrees and $10 \%$ in the 90 degrees direction. This allows the application of one single variable while maintaining the design guidelines that require at least $10 \%$ fibers in each ply direction. Here, it is again emphasized that the chosen test case is representative for a typical problem in aircraft design, and that the geometry, the load case, the material and the failure criteria could easily be changed. Moreover, further optimization is possible by allowing the variation of the stacking sequence. This can lead to more efficient load distribution among skin and stiffeners and result in configurations with lower weight and cost.

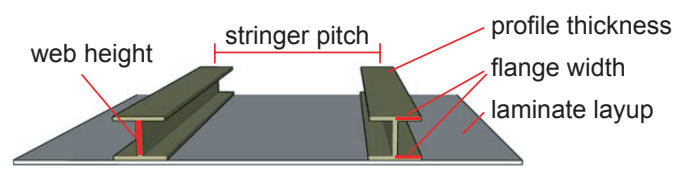

Figure 5: Generic skin/stringer element

For the sake of simplicity, any depreciation factors and cost adjustments were neglected (i.e. $\alpha_{1}=\alpha_{2}=\alpha_{3}=1$ ), and the same equipment and operator rates for in-production and in-service inspection were applied. The prescribed probability of detection was set to $95 \%$.

Here, five regular inspections during the lifetime of the aircraft were assumed. Therefore, the objective function results in

$$
D O C=C_{\text {man }}+C_{n d t, p r o d}+5 C_{n d t, s e r v}+p W
$$

The attention is drawn to the quantification of the weight penalty. One can imagine that the cost/weight tradeoff is dependent on factors such as accumulated flight distance during aircraft life, engine specific fuel burn, thrust level during flights, environmental impact penalties or fuel price. An objective determination of $p$ is difficult, as it may vary from customer to customer, the application (civil, cargo, military version of the same plane), whether it is optimized for additional payload, fuel saving or additional passengers, and the way the customer evaluates his product. The literature proposes a weight penalty between $€ 45 / \mathrm{kg}$ and $€ 380 / \mathrm{kg}$. Own research, based on the fuel consumption of an airliner in the A330 class and today's 
fuel price, reveals a different picture. In this specific case, the weight penalty $p$ would result in about $€ 1840$ lifetime fuel burn cost per kg aircraft mass. Structural mass reduction of one kilogram might also involve a reduction of the gross weight that is significantly higher, as the weight of engines and other systems can be lowered. And when taking the revenue of an additional passenger into account, this value could be one order of magnitude higher. It was decided to apply a value of $€ 1500 / \mathrm{kg}$ to the case study as a compromise between values from the literature and our own calculations.

The FE model was built up in ABAQUS/CAE according to Figure 5. It was constrained by periodical boundaries along the sides and meshed with shell elements of the type S4R. The introduction of the compressive load was realized by means of two rigid bodies, simulating the adjacent frame structure. The adhesive bonds were approximated by tie constraints, thus preventing any separation of skin and stringers. Two failure criteria were checked during the FE analysis. First, the maximum strain values (MSTRN) were sought in all integration points of the skin and stringer nodes, and second, the eigenvalues of a linear perturbation analysis was calculated. The results from both criteria were fed back to the optimization solver. Rather high loads were applied to this structure $(2.5 \mathrm{kN}$ per mm width) in order to obtain comparable failure loads for buckling and strain. As can be seen in the results, the model consisted of rather stocky stiffeners. The maximum strain criterion was set to $0.005,-0.004$ and 0.008 for tension, compression and shear, respectively.

Regarding the NDT model, the structure was divided into different features, such as the skin laminate, further two stringers with two flange laminates, one web laminate and four radii each; the two adhesive bonds between skin and stringer were two other features.

The adjustment of the allowables due to porosity was estimated according to Equation (6). The value of $B=-3.14$ was adopted from Uhl et al. [23] who performed a number of test series for a 16 ply quasi-isotropic carbon/epoxy laminate. The void content $v_{p}$ as function of the thickness was required as input. The approximate function $v_{p}=0.0025 t$ was extracted from the figures presented by Lee and Soutis [26]. In their work they studied the void content with respect to the thickness of the specimens.

In this first attempt, the strength reduction due to flaws of a given size $f$ was based on the work done by Soutis et al. [30], from which the value of $\hat{\sigma}_{f}(f)=$ $\hat{\sigma}_{0}\left(1-\frac{12.4}{444}(f-6)\right)$ was extracted. Parameter $\hat{\sigma}_{0}$ refers to the strength of a coupon test with $6 \mathrm{~mm}$ hole size.

The skin/stringer panel was first optimized for a series of weight penalties, thus resulting in a parameter study covering the whole range from pure cost optimization $(p=0)$ to pure weight optimization $(p=\infty)$. Therefrom, three configurations were chosen as the starting points for the subsequent optimizations: A low-cost panel (a), a cost/weight optimized panel with a fuel penalty of $€ 1500 / \mathrm{kg}$ (b), and a low-weight panel (c). All three baselines were obtained without the activation of the NDT module. Then, the behavior of each configuration was investigated when varying 
the quality level and laminate thicknesses while the geometry was kept constant. Finally, the influence of the $P O D$ on the direct operating cost was examined.

\section{Results and discussion}

The first part of this section regards the parameter study done without activation of the NDT model. Hence, the results were obtained by optimizing a skin/stringer element with an objective function given as $D O C=C_{m a n}+p W$ and the weight penalty $p$ varying between 0 and infinity. The results are shown in Figure 6 .

A distinctive change in configuration can be seen at a weight penalty of $€ 1000$ $10^{\prime} 000 / \mathrm{kg}$. To the left of this change, a low-cost solution is preferable. There, the skin/stringer panel consists of a thick skin and bulky stiffeners with maximum pitch. Since fewer stringers are proposed in the low-cost solution, manufacturing costs can be saved in spite of the higher cost per stiffener. Further, the buckling constraint (but not the maximum strain constraint) is active. Hence, the structure is not performing optimally, and some more cost saving could be possible by enlarging the design space. To the right, the low-weight configuration comprises a densely stiffened, thin skin with rather fine stringers where both structural constraints were active. Three design solutions were now chosen as the starting point for the subsequent investigation, see Table 1. Properties marked with asterisks were kept constant in the subsequent investigation.

Table 1: Starting points for the investigation of different laminate qualities.

\begin{tabular}{lrrrrr}
\hline configuration & low-cost & intermediate & low-weight & lower & upper \\
$p$ [in $€ / \mathrm{kg}]$ & 0 & 1500 & $\infty$ & limit & limit \\
\hline skin thickness $[\mathrm{mm}]$ & 8.00 & 7.96 & 5.31 & 0.26 & 8 \\
stringer thickness [mm] & 8.88 & 2.53 & 3.77 & 1 & 25 \\
stringer foot* $[\mathrm{mm}]$ & 24.86 & 14.50 & 5.00 & 5 & 50 \\
web height* $[\mathrm{mm}]$ & 20.96 & 22.80 & 20.00 & 20 & 60 \\
stringer pitch* $[\mathrm{mm}]$ & 300.00 & 184.91 & 100.13 & 100 & 300 \\
\hline
\end{tabular}

\section{Quality optimization of the low-cost panel $(p=€ 0 / k g)$}

First, the cost optimized panel with a stringer pitch of $300 \mathrm{~mm}$ was investigated. This panel was optimized for a series of flaw sizes, varying from 2 to $20 \mathrm{~mm}$. The results are compiled in Table 2, where a flaw size of $6 \mathrm{~mm}$ represents the baseline.

As can be seen, the variation of the laminate quality has a considerable impact on the objective function $D O C$. At a flaw size of $2 \mathrm{~mm}$, the laminates have to be scanned much denser in order to guarantee a probability of detection of $95 \%$. The result is an NDT cost that is 2.74 times the baseline and a DOC that is $46 \%$ higher than the baseline. It also can be seen that only the buckling constraint is active for 

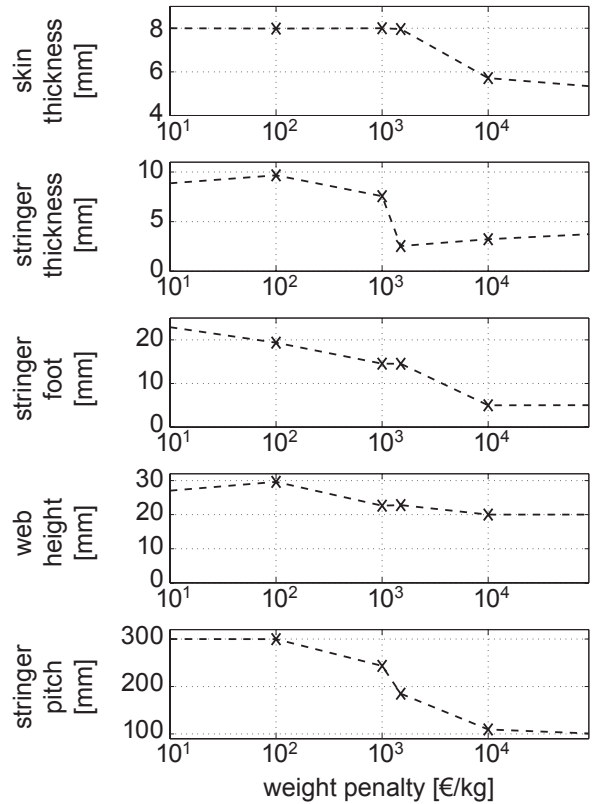

Figure 6: Results from the preparatory parameter study

small flaw sizes. As the buckling load is independent of the strength of the material, no weight reduction is possible in this case.

The cost for inspection $C_{n d t}$ decreases when the flaw size increases, as the scan time is reduced. The optimum configuration can be found at a flaw size of $16.3 \mathrm{~mm}$. There, the active constraint switches from buckling to maximum strain and the objective function $D O C$ is $14 \%$ lower than the one of the baseline. Any further increase of the flaw size is disadvantageous, as the impact of the manufacturing cost due to additional material (to resist material failure) is too high.

Table 2: Quality optimization of the low-cost panel $(p=€ 0 / \mathrm{kg})$

\begin{tabular}{lrrrrrr}
\hline flaw size & $2 \mathrm{~mm}$ & $6 \mathrm{~mm}$ & $10 \mathrm{~mm}$ & $14 \mathrm{~mm}$ & $16.3 \mathrm{~mm}$ & $20 \mathrm{~mm}$ \\
\hline DOC & 1.46 & 1.00 & 0.91 & 0.87 & 0.86 & 1.00 \\
weight & 1.00 & 1.00 & 1.00 & 1.00 & 1.00 & 1.14 \\
$C_{\text {ndt }}$ & 2.74 & 1.00 & 0.66 & 0.51 & 0.46 & 0.46 \\
$C_{\text {man }}$ & 1.00 & 1.00 & 1.00 & 1.00 & 1.00 & 1.19 \\
buckling & 1.00 & 1.00 & 1.00 & 1.00 & 1.00 & 0.92 \\
MSTRN & 0.58 & 0.58 & 0.67 & 0.81 & 0.92 & 1.00 \\
\hline
\end{tabular}




\section{Quality optimization of the intermediate panel $(p=€ 1500 / \mathrm{kg})$}

Second, an intermediate panel design with $€ 1500 / \mathrm{kg}$ was investigated. Similar to the preceding example, the cost of this panel was recalculated adding the cost for in-production and in-service inspection with a standard flaw size of $6 \mathrm{~mm}$. Then the panel was optimized for a series of flaw sizes, see Table 3.

As for the previous case, the objective function is the highest for the smallest flaw size $(2 \mathrm{~mm})$ due to the high NDT cost. As the panel thicknesses are constrained by the active buckling condition, no weight or cost saving is possible for this laminate quality.

As long as buckling governs the design, an increased flaw size mainly results in lower NDT cost while the weight and the manufacturing cost remain the same. At a flaw size of $12.4 \mathrm{~mm}$, the maximum strain constraints also becomes active, and the $D O C$ achieves its minimum at $96 \%$ compared to the baseline. Any increase of flaw size results in increased weight and manufacturing cost, thus forcing the objective function up. The stringer cross-sections are very thick at flaw sizes of $14 \mathrm{~mm}$ and $20 \mathrm{~mm}$, due to the reduced strength. As the cross-sections are extraordinarily thickened, more scanning is necessary in order to guarantee a $P O D$ of $95 \%$. The result is an increase of NDT cost, despite the lower laminate quality.

Table 3: Quality optimization of the intermediate panel $(p=€ 1500 / \mathrm{kg})$

\begin{tabular}{lrrrrrr}
\hline flaw size & $2 \mathrm{~mm}$ & $6 \mathrm{~mm}$ & $10 \mathrm{~mm}$ & $12.4 \mathrm{~mm}$ & $14 \mathrm{~mm}$ & $20 \mathrm{~mm}$ \\
\hline DOC & 1.16 & 1.00 & 0.97 & 0.96 & 1.00 & 1.37 \\
weight & 1.00 & 1.00 & 1.00 & 1.00 & 1.05 & 1.46 \\
$C_{n d t}$ & 2.38 & 1.00 & 0.72 & 0.63 & 0.65 & 0.68 \\
$C_{\text {man }}$ & 1.00 & 1.00 & 1.00 & 1.00 & 1.05 & 1.46 \\
buckling & 1.00 & 1.00 & 1.00 & 1.00 & 0.95 & 0.50 \\
MSTRN & 0.78 & 0.77 & 0.90 & 1.00 & 1.00 & 1.00 \\
\hline
\end{tabular}

\section{Quality optimization of the low-weight panel $(p=€ \infty / k g)$}

Third, a weight optimized panel was exposed to the inclusion of NDT cost. In Table 4, corresponding results are shown. As the manufacturing and non-destructive testing costs are negligible in this case, the $D O C$ only depends on the weight. As anticipated, the optimum laminate quality is found at very small flaw sizes, and both structural constraints are active.

\section{Variation of the POD}

Non-destructive testing never reaches a $P O D$ of $100 \%$. In practice, an artificial limit is set to this value, representing a measure of trustworthiness for the scanning method. The effect of the $P O D$ on the results is now investigated. 
Table 4: Quality optimization of the low-weight panel $(p=€ \infty / \mathrm{kg})$

\begin{tabular}{lrrrrr}
\hline flaw size & $2 \mathrm{~mm}$ & $6 \mathrm{~mm}$ & $10 \mathrm{~mm}$ & $14 \mathrm{~mm}$ & $20 \mathrm{~mm}$ \\
\hline DOC & 0.99 & 1.00 & 1.13 & 1.36 & 1.93 \\
weight & 0.99 & 1.00 & 1.13 & 1.36 & 1.93 \\
$C_{n d t}$ & 2.07 & 1.00 & 0.85 & 0.75 & 0.74 \\
$C_{\text {man }}$ & 1.00 & 1.00 & 1.15 & 1.24 & 1.36 \\
buckling & 1.00 & 1.00 & 0.82 & 0.48 & 0.22 \\
MSTRN & 1.00 & 0.98 & 1.00 & 1.00 & 1.00 \\
\hline
\end{tabular}

The results shown in Table 5 apply for the intermediate panel with a weight penalty $p=€ 1500 / \mathrm{kg}$. As expected, the quality of laminate is not influenced by the $P O D$, and the optimal design solutions are the same in all cases. On the other hand, an increase in NDT cost and thus an increase in the objective function can be seen for higher probabilities. This is the cost one has to account for when a higher NDT reliability is desired.

Table 5: Optimization with variable $P O D$ for the intermediate panel $(p=€ 1500 / k g)$

\begin{tabular}{lrrrrrr}
\hline POD & $80 \%$ & $85 \%$ & $90 \%$ & $95 \%$ & $97.5 \%$ & $99 \%$ \\
\hline DOC & 0.97 & 0.98 & 0.99 & 1.00 & 1.01 & 1.03 \\
weight & 1.00 & 1.00 & 1.00 & 1.00 & 1.00 & 1.00 \\
$C_{n d t}$ & 0.76 & 0.81 & 0.89 & 1.00 & 1.11 & 1.24 \\
$C_{\text {man }}$ & 1.00 & 1.00 & 1.00 & 1.00 & 1.00 & 1.00 \\
buckling & 1.00 & 1.00 & 1.00 & 1.00 & 1.00 & 1.00 \\
MSTRN & 0.78 & 0.78 & 0.78 & 0.78 & 0.78 & 0.78 \\
\hline
\end{tabular}

\section{Conclusions}

A framework for the cost/weight optimization of composite structures under consideration of a variable laminate quality was proposed. A generic skin/stringer element was adopted in a case study, and a parameter study was performed in which the permissible flaw size was varied.

An objective function was selected that included manufacturing cost, in-production and in-service inspection and a weight penalty. Starting from a standard flaw size, the effects of the laminate quality on the objective function were investigated. The optimal flaw size depended on the structure, the material data, the load case and the weight penalty. Hence, it was shown that cost savings are possible by revising the laminate quality already in the design phase. The conclusions (suggesting that 
the permissible flaw size does not have to be $6 \mathrm{~mm}$ ) should be viewed as specific to the assumptions and analysis made and they may not be valid when, for example, fatigue is taken into account. Further work is needed in this area before any general conclusions can be made.

This optimization framework may also be applicable outside the aerospace industry. As an example, one could consider its implementation for the production of rotor blades for windmills.

\section{$6 \quad$ Future work}

The proposed optimization framework represents a first approach to include quality management aspects in the design process. More work, however, has to be done in order to improve the NDT cost and the NDT strength reduction models. First, the difference between in-production and in-service testing needs to be more elaborate by applying different scanning techniques and overhead adjustments. Second, the strength reduction should be expressed as a function of the stacking sequence, the material properties and the manufacturing technique. Third, one should investigate whether a stiffness reduction due to porosity should be included in the structural model. Fourth, the inspection interval should be adapted to the stress level and the structural function of each member. And finally, a probabilistic damage model should be included to capture possible damage and repair of a structural member. This could include a more detailed ultrasonic analysis, which in turn causes a cost increase.

\section{Acknowledgments}

This work is part of the European Framework Program 6, project ALCAS, AIP4-CT2003-516092. Special thanks go to Alfgam AB for the use of Xopt and to Galorath International for the use of the cost estimation package SEER-MFG.

\section{Bibliography}

[1] T. S. Geiger and D. M. Dilts. Automated design-to-cost: Integrating costing into the design decision. Computer Aided Design, 28(6-7):423-438, 1996.

[2] B. Heinmuller and D. M. Dilts. Automated design-to-cost: Application in the aerospace industry. In Proceedings - Annual Meeting of the Decision Sciences Institute, volume 3, pages 1227-1229, 1997.

[3] C. Kassapoglou. Simultaneous cost and weight minimization of composite-stiffened panels under compression and shear. Composites Part A: Applied Science and Manufacturing, 28(5):419-435, 1997.

[4] C. Kassapoglou. Minimum cost and weight design of fuselage frames. part a: Design constraints and manufacturing process characteristics. Composites Part A: Applied Science and Manufacturing, 30(7):887-894, 1999. 
[5] C. Kassapoglou. Minimum cost and weight design of fuselage frames. part b: Cost considerations, optimization, and results. Composites Part A: Applied Science and Manufacturing, 30(7):895-904, 1999.

[6] W. J. Marx, D. N. Mavris, and D. P. Schrage. A knowledge-based system integrated with numerical analysis tools for aircraft life-cycle design. Artificial Intelligence for Engineering Design, Analysis and Manufacturing, 12:211-229, 1998.

[7] K. Gantois and A. J. Morris. The multi-disciplinary design of a large-scale civil aircraft wing taking account of manufacturing costs. Structural and Multidisciplinary Optimization, 28:31-46, 2004.

[8] J. Scanlan, A. Rao, C. Bru, P. Hale, and R. Marsh. Datum project: Cost estimating environment for support of aerospace design decision making. Journal of Aircraft, 43(4):1022-1028, 2006.

[9] C. H. Park, W. I. Lee, and A. Vautrin. Simultaneous optimization of composite structures considering mechanical performance and manufacturing cost. Composite Structures, 65(1):117-127, 2004.

[10] M. S. Edke and K. H. Chang. Shape optimization of heavy load carrying components for structural performance and manufacturing cost. Structural and Multidisciplinary Optimization, 31(5):344-354, 2006.

[11] R. Curran, G. Gomis, S. Castagne, J. Butterfield, T. Edgar, C. Higgins, and C. McKeever. Integrated digital design for manufacture for reduced life cycle cost. International Journal of Production Economics, 109(1-2):27-40, 2007.

[12] D. Kelly, K. Wang, and S. Dutton. A guided tradeoff for cost and weight for generating optimal conceptual designs. In Collection of Technical Papers AIAA/ASME/ASCE/AHS/ASC Structures, Structural Dynamics and Materials Conference, volume 2, pages 894-904, 2003.

[13] K. Wang, D. Kelly, and S. Dutton. Multi-objective optimisation of composite aerospace structures. Composite Structures, 57(1):141-148, 2002.

[14] R. Curran, A. Rothwell, and S. Castagne. A numerical method for costweight optimisation of stringer-skin panels. In Collection of Technical Papers AIAA/ASME/ASCE/AHS/ASC Structures, Structural Dynamics and Materials Conference, volume 7, pages 5262-5277, 2004.

[15] M. Kaufmann, D. Zenkert, and P. Wennhage. Integrated cost/weight optimization of composite skin/stringer elements. In Takashi Ishikawa, editor, Proceedings of 16th International Conference on Composite Materials, Kyoto, 2007.

[16] J. Roskam. Airplane Design Part VIII: Airplane Cost Estimation Design Development and Manufacturing and Operating. Darcorporation, 1990.

[17] W. E. Schmid. Costs involved in non-destructive testing of materials. Maschinenbau, 16(19/20):519-522, 1937.

[18] D. J. Hagemaier. Cost benefits of nondestructive testing in aircraft maintenance. Materials Evaluation, 46(10):1272-1284, 1988. 
[19] D. J. Hagemaier. Effective implementation of NDT into aircraft design, fabrication, and service. Materials Evaluation, 46(7):851-868, 1988.

[20] A. A. Kale, R. T. Haftka, and B. V. Sankar. Tradeoff of structural weight and inspection cost in reliability based optimization using multiple inspection types. In Collection of Technical Papers - 10th AIAA/ISSMO Multidisciplinary Analysis and Optimization Conference, volume 2, pages 1162-1173, 2004.

[21] A. Niazi, J. S. Dai, S. Balabani, and L. Seneviratne. Product cost estimation: Technique classification and methodology review. Journal of Manufacturing Science and Engineering, Transactions of the ASME, 128(2):563-575, 2006.

[22] N. C. W. Judd and W. W. Wright. Voids and their effects on the mechanical properties of composites - an appraisal. SAMPE Journal, 14(1):10-14, 1978.

[23] K. M. Uhl, B. Lucht, H. Jeong, and D. K. Hsu. Mechanical strength degradation of graphite fiber reinforced thermoset composites due to porosity. Review of Progress in Quantitative Nondestructive Evaluation, (7B):1075-1082, Year $=1988$.

[24] A. M. Rubin and K. L. Jerina. Effect of porosity on elastic constants of representative aircraft laminates. Journal of Advanced Materials, 25(4):21-30, 1994.

[25] M. L. Costa, S. F. M. Almeida, and M. C. Rezende. Critical void content for polymer composite laminates. AIAA Journal, 43(6):1336-1341, 2005.

[26] J. Lee and C. Soutis. Thickness effect on the compressive strength of t800/924c carbon fibre-epoxy laminates. Composites Part A: Applied Science and Manufacturing, 36(2 SPEC ISS).

[27] J. Lee and C. Soutis. A study on the compressive strength of thick carbon fibre-epoxy laminates. Composites Science and Technology, 67(10):2015-2026.

[28] G. A. Georgiou. Pod curves, their derivation, applications and limitations. Insight: Non-Destructive Testing and Condition Monitoring, 49(7):409-414, 2007.

[29] C. K. Huang and K. Y. Lin. A method for reliability assessment of aircraft structures subject to accidental damage. In Collection of Technical Papers AIAA/ASME/ASCE/AHS/ASC Structures, Structural Dynamics and Materials Conference.

[30] C. Soutis, F. C. Smith, and F. L. Matthews. Predicting the compressive engineering performance of carbon fibre-reinforced plastics. Composites Part A: Applied Science and Manufacturing, 31(6):531-536, 2000.

[31] K. Svanberg. Method of moving asymptotes - a new method for structural optimization. Journal for Numerical Methods in Engineering, 24(2):359-373, 1987.

[32] K. Svanberg. A globally convergent version of MMA without linesearch. In Proceedings of the First World Congress of Structural and Multidisciplinary Optimization, Goslar (Germany), pages 9-16, May/June 1995. 